Bei beginnender Tabes sieht man unter spezifischer Therapie mit Jod, Quecksilber und Salvarsan noch am häufigsten einen Erfolg. Auch Massage, Faradisation, Heißluftduschen, Exzision mit nachfolgender Kauterisation und Quarzlichtbestrahlungen werden empfohlen und können gelegentlich helfen. Socin empfiehlt, die Ulzera nach vorhergehender Reinigung mit Jodtinktur und Alkohol mit einer dicken Paste aus 10\%iger Zinkchloratlösung und Zinkoxydpulver zu plombieren und einen hydrophilen wasserdichten Verband anzıllegen. Sehr häufig versagen aber alle diese therapeutischen Maßnalımen, und der Arzt steht diesen hartnäckigen Geschwüren hilf- und ratlos gegenüber.

Im vergangenen Jahre kamen hier 2 Fälle von Mal perforant zur Beobachtung, die bis dalin jeder Therapie getrotzt latten; beide Patienten hatten eine Tabes.

1. H. H., 46jälıriger Schmied, vor 10 Jahren Lues. Bald nach der Infektion 3 Sclinilerkuren, in den letzten beiden Jahren 3 gute kombinierte Quecksilber-Silbersalvarsankuren, daneben dauernd Jodkali. Seit 3 Jahren Geschwüre an der Fußsohle, die an sich nur wenig Schmerzen machen, aber durch die stinkende Eiterabsonderung und gelegentlichen Entzündungen den Patienten bei der Arbeit arg behindern. Vor einem Jahre sind beide großen Zehen mit dem Kapitulunı des Mittelfußknochens entfernt; unter dem Köpfchen des 3. Mittelfußknochens verblieb an beiden Fußsohlen ein talergroßes typisches Mal perforant bestehen, das trotz spezifischer und Salbenbehandlung, Wechselbäder, Blaulichtbestrahlungen und Bettruhe nicht zur Heilung gebracht werden konnte.

4. X. 1920 Bestrahlung.

2. H. D., Lues ignota. Seit Ende 1919 wegen Tabes mit schweren Blasenlälmungen in spezifischer Behandlung; macht Anfang 1920 eine Jodipin-Silbersalvarsan-Hg-Kur durch. Am Sclıluß der Kur stellen sich Schnerzen an beiden Fersen ein; innerhalb weniger Wochen entwickeln sich unter beiden Fußsohlen Uizera, welche die Größe eines Fünfmarkstückes erreichen und die Sehnen freilegen. D. kann nur mit größter Mühe gehen, muß seiıe Berufstätigkeit bedeutend einschränken. Lokalbehandlung und eine Silbersalvarsankur September bis Oktober 1920 bringen keine Besserung; die Beschwerden werden immer größer.

12. XI. 1920 Bestrahlung.

$D_{i 1}$ in beiden Fällen die üblichen Behandlungsmethoden sehr lange ohne Erfolg geblieben waren, wurden die Patienten der Röntgenbehandlung unterzogen. Bestrahlt wurde mit MüllerSiederöhre mit $3 \mathrm{~mm} \mathrm{A.} \mathrm{F.,} \mathrm{pro} \mathrm{Sitzung} \mathrm{wurden} 9 \mathrm{X}$ verabfolgt, nach jeder Bestrahlung 14 Tage Pause, dann erneute Bestrahlung. MeBmethode nach $\mathrm{H}$ ans $M$ eyer (Bremeil). Von jeder allgemeinen und lokalen Behandlung wurde abgeselıen. Mit einem indifferenten Salbenschutzverband gingen die Patienten ihrer gewohnten Beschäftigung nlach. Der Erfolg war überraschend, bci $H$. H. waren die Geschwüre nach 2 Volldosen, bei H. D. nach einer Volldosis völlig abgeheilt. Beide Patienten sind heute noch nach einem halben bzw. nach einem viertel Jahr rezidivfrei und voll erwerbsfähig.

Eine Erklïirung über die Wirkung der Röntgenstrahlen bei Mal perforant vermögen wir nicht zu geben; walırsclieinlich handelt es sich um eine Reizwirkung der Strahlen auf das in seiner Ernährung geschädigte Gewebe und die sclılaffen Granulationen des Wundgrullides.

Aus der Abteilung für Strahlenbehandlung der Städtischen Krankenanstalten in Essen. (Leiter: Prof. Fr. Bering.)

\title{
Röntgenbehandlung des Mal perforant du pied.
}

\author{
Von Dr. L. Kleinschmidt, Oberarzt der Hautklinik.
}

Die unter dem Namen Mal perforant du pied bekanılıten Geschwüre habenl ihren Sitz an der Fußsohle, an der Stelle des stärksten Druckes, gewöhnlich am Groß- und Kleinzehenballen und an der Ferse; bei Fußdeformitäten, Plattfüßen und Klumpfüßen auch an anderen Stellen. Die fingerkuppen- bis pfenniggroßen, äußerst chronisch verlaufenden. kraterförmigen Ulzera mit ihrem schmierigen Grunde und ihren steil abfallenden Wundrändern sind von einem hyperkeratotischen Wall umgeben und sondern einen dünnflüssigen, übelriechenden Eiter $a b$ Sie entwickeln sich gewöhnlich unter schmerzhaften Schwielen und Hïhnerauren, die durch Gewebsabszedierung unterminiert werden und kraterförmig einschmelzen. Bei schlechter oder erfolgloser Behandlung können sie bis auf die Sehnen, Knochen und Gelenke reichen, zu Phlegmonen und weitgehenden Zerstörungen führen.

Die Aetiologie des Mal perforant ist nicht einlıeitlich, eine Reilie von zentraien, spinaen und peripherischen Nervenleiden wird mit der Erkrankung in ursächlichen Zusilmmenhang gebracht. Am häufigsten sieht man sie bei Tabes, oft bei Syringonyelte, auch bei Rïckenmarksläsionen durch Wirbelverletzungen und -tumoren, bei Neuritis, Nervengeschwülsten und -traumen, Diabetes, Arteriosklerose und Lepra.

Bei der Mannigfaltigkeit der Grundkranklheiten ist eine einlheitliche kausale Therapie von vornherein wenig aussichtsvoll. Aeußer Schädlichkeiten, die einen dauernden Druck verursachen, wie schlechtsitzende Scluthe, sind nach Möglichkeit zu vermeiden. Bettruhe ist gewiß gut, aber nur in den wenigsten Fällen durchzuführen und führt schließliclı doch nicht zum Ziele. Chirurgische Eingriffe sind sehr eingreifend, verstümnelnd und z. B. an der Ferse auch schlecht ausführbar. 